\title{
Self-induced photorefractive spatial frequency filter
}

\author{
G. Möbus, B. Schmidt, and H. J. Tiziani \\ Institute of Applied Optics, University of Stuttgart, 7000 Stuttgart 80, Germany
}

Received June 18, 1990; accepted July 11, 1990

\begin{abstract}
$\mathrm{A} \mathrm{Bi}_{12} \mathrm{SiO}_{20}$ crystal, cut in the transverse orientation as for holographic applications, is used as a spatial filtering device in the Fourier plane of a coherent single-beam optical processor with $\lambda=633 \mathrm{~nm}$. Frequency selection is obtained by adjusting a polarizing compensator to suppress or to select some part of the spectrum, which is characterized by a certain ellipticity of polarization, depending on its intensity.
\end{abstract}

\section{INTRODUCTION}

Photorefractive crystals change their refractive index under nonhomogenous illumination and can therefore be used as a light-intensity recording medium. ${ }^{1,2} \quad \mathrm{Bi}_{12} \mathrm{SiO}_{20}$ (BSO) crystals have been used since the early 1970's as spatial light-modulating, holographic-recording, and nonlinear-beam-coupling media with applications in correlation and spatial frequency filtering. ${ }^{3-7}$ Some papers deal with techniques for obtaining edge enhancement ${ }^{8,9}$ or defect enhancement in periodical structures ${ }^{10}$; all these studies use the effect of diffraction-efficiency dependence on the object-to-reference-beam ratio in holographic processes or grating-erasure methods.

In each of these applications a short-wavelength light source (typically a 514- $\mathrm{nm} \mathrm{Ar}^{+}$laser) is used to address the crystal, while readout is obtained either by using the same wavelength (as in degenerate four-wave mixing and two-beam coupling) or with nondestructive red (e.g., $\mathrm{He}-\mathrm{Ne}$ laser) light when the absorption coefficient in BSO is much smaller, as it is for $\lambda=514 \mathrm{~nm}$.

When inserted into the Fourier plane of a coherent optical setup, the crystal is, however, affected by the red light. The high intensities of the zero and higher diffraction orders, because of periodicities in the input, lead to a drastic conductivity increase of those highly illuminated areas compared with the remaining areas of the BSO crystal. Experiments showed clearly that the laser-light-induced conductivity is sufficient to cancel the externally applied electrical field. With polarization optics these diffractions orders can therefore be selected or suppressed, depending on their intensity, the orientation of the polarizers, and the (optional) quarter-wave plate.

The result is that the filter function is generated directly by the Fourier spectrum of the input object and therefore adapts to different objects within fractions of a second. Furthermore, no adjustment for the filter is needed because writing and readout are performed by the same beam, which is of course always aligned with itself. By means of different orientations of the polarizing components, several filter functions and applications can be accomplished:

Edge enhancement by suppression of the zero order;

Zernike-like phase contrast with selectable contrast sign by phase shift and reduction of the zero order;
Error detection on periodic objects by suppression of the diffraction peaks;

Removal of unperiodic structures such as speckle noise or other errors by selection of only the diffraction peaks.

\section{THEORY}

In our experiments we used a BSO crystal with a transversally applied electrical field, as is common for holographic setups. ${ }^{1}$ With no applied field the crystal exhibits only optical activity, but when the field is switched on the crystal becomes birefringent, with its main axes lying on the diagonals of the crystal (see Fig. 1).

As is mentioned above, the high-intensity diffraction orders cause a highly conducting (compared to the unaffected regions) area around themselves in the BSO crystal, so that the strength of the externally applied electrical field, which causes the crystal to become birefringent, is locally decreased. Ohm's law can be written in terms of the electrical field $E$, the conductivity $\sigma$, and the current flow $j$ as

$$
E=j / \sigma .
$$

The conductivity of the BSO is the sum of its dark conductivity and photoconductivity $\sigma_{d}$ and $\sigma_{p}$, respectively:

$$
\sigma=\sigma_{d}+\sigma_{p}=\sigma_{d} / \eta I,
$$

with light intensity $I$ and photoconductivity coefficient $\eta$, which changes with wavelength. For $\lambda=633 \mathrm{~nm}$ these parameters are

$$
\begin{aligned}
\sigma_{d} & =1.6 \times 10^{-15} \Omega^{-1} \mathrm{~cm}^{-1}, \\
\eta & =3.7 \times 10^{-10}{\mathrm{~cm} \Omega^{-1} \mathrm{~W}^{-1} .} .
\end{aligned}
$$

Considering the typical power densities in the Fourier plane for diffraction orders of periodical structures of $0.1-10 \mathrm{~W} \mathrm{~cm}^{-1}$, one can easily estimate that the conductivity in the spot-illuminated areas is more than 20,000 -fold higher than in the dark regions. This means that the whole applied voltage drops across the nonilluminated regions of the crystal, leading to an almost completely canceled electrical field for the diffraction spots. This effect can be used to generate a self-induced Fourierplane Filter by converting the refractive-index changes 


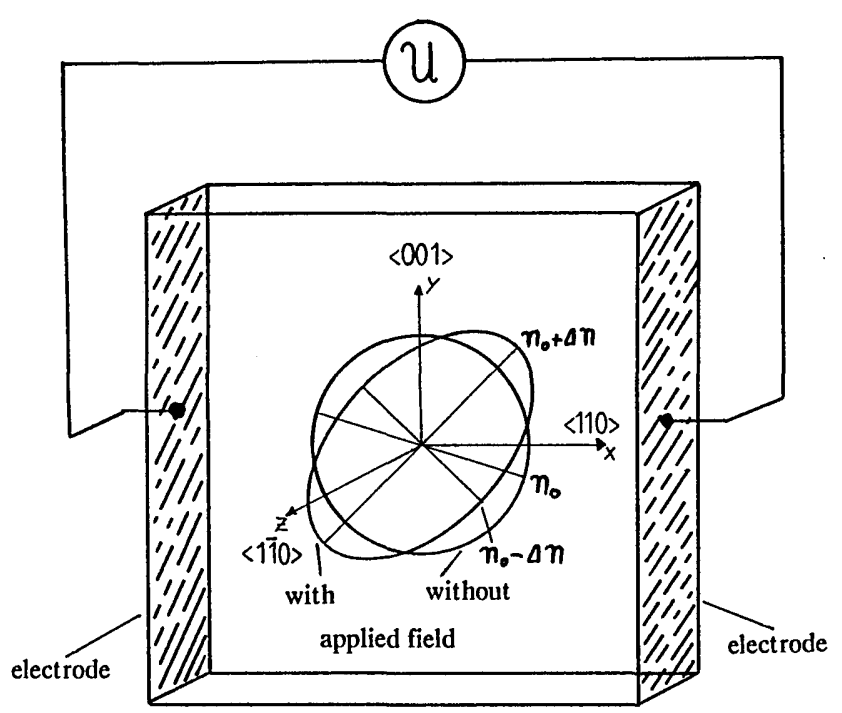

Fig. 1. Orientation and indicatrix of the BSO crystal with and without applied electrical field.

caused by the varying electrical field strength into amplitude or phase changes by means of polarization optics.

The refractive-index change of the main axes in the BSO crystal (Fig. 1), when an electrical field is applied to it, is given by

$$
n_{ \pm}=n_{0} \pm 1 / 2 n_{0}{ }^{3} r_{41} E \text {. }
$$

To calculate the phase shift and the amplitude-filtering effects of the setup, we applied the Jones formalism for the representation of the polarization states in transparent media. ${ }^{11,12}$ The matrix for a BSO plane-parallel plate with its main axes oriented along the diagonals of the $x-y$-coordinate axes is

$$
M_{\mathrm{BSO}}=\left[\begin{array}{cc}
\cos (d r g) & \left(-\rho+i g_{45}\right) \frac{\sin (d r g)}{r g} \\
\left(\rho+i g_{45}\right) \frac{\sin (d r g)}{r g} & \cos (d r g)
\end{array}\right],
$$

with

$r g=\left(\rho^{2}+g_{45}^{2}\right)^{1 / 2}$, $g_{45}=(\pi / \lambda) n_{0}^{3} r_{41} E$,

$\rho=23.5^{\circ} \mathrm{mm}^{-1}$ (gyration constant of BSO at $\lambda=$ $633 \mathrm{~nm})$

$r_{41}=3.7 \times 10^{-10} \mathrm{~cm}^{-1} \mathrm{~V}^{-1}$ (electro-optic coefficient), ${ }^{13}$

$d=3 \mathrm{~mm}$ (crystal thickness in the direction of the axis),

$n_{0}=2.53$ (refractive index of BSO without the applied electrical field $E$ ).

Conversion of the refractive-index changes in the crystal into amplitude and phase variations requires readout with linearly polarized light. Since the writing and readout lights are identical, the input light must already be properly polarized, which is accomplished by a linear polarizer in front of the BSO crystal. After passing the crystal the light will be more or less elliptically polarized, depending on its intensity. With a quarter-wave plate it is possible to convert any elliptically polarized light back into linearly polarized light, which in turn can be blocked by a suitably oriented analyzer. Therefore we obtain a filter that is able to block light with an arbitrary intensity in the Fourier plane. The selectivity of the filter is quite poor, but because of the immense intensity differences in the power spectrum it is easy to separate the highintensity diffraction orders from the remaining part of the spectrum.

Figure 2 shows the optical setup that we used in our experiments. To overcome the problems of vignetting and for more flexibility, we chose the converging-beam optical processor. ${ }^{14}$ To increase the total conductivity of the crystal a white-light source, providing incoherent background illumination, could optionally be used. This would cause the light that carries nonperiodic information to be more uniformly elliptically polarized so it could be blocked by the combination of a quarter-wave plate and an analyzer for suppression of periodic errors. A constantbackground illumination also reduces the size of the highly conducting spots, which can then be more sharply selected.

As long as the periodic or the nonperiodic part of the spectrum is totally suppressed by the filter, it is not necessary to consider phase changes between these two spectral parts that are due to the different optical path lengths, which are caused by the varying birefringence. However, if the filter is adjusted to transmit both spectral parts,

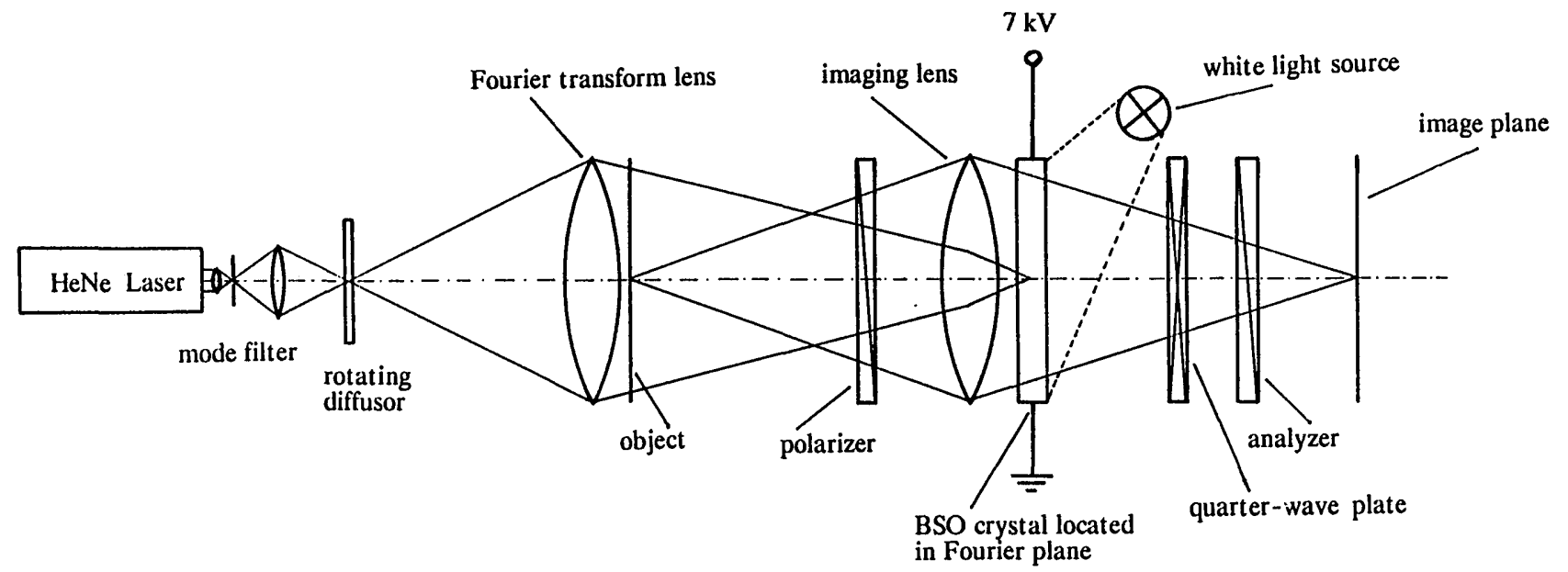

Fig. 2. Optical setup. 


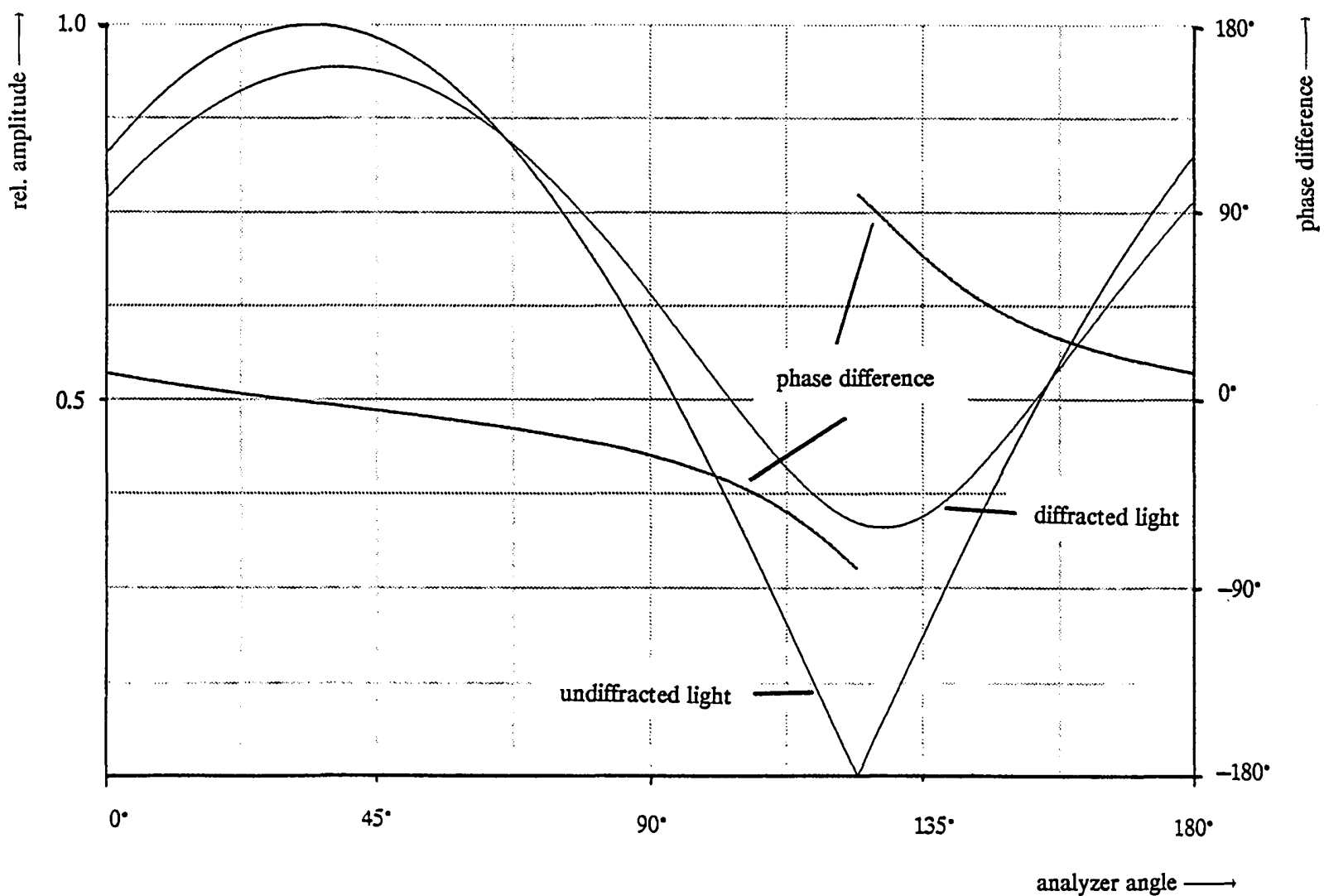

Fig. 3. Dependence of amplitude and phase transmission function of the BSO filter on the analyzer orientation.

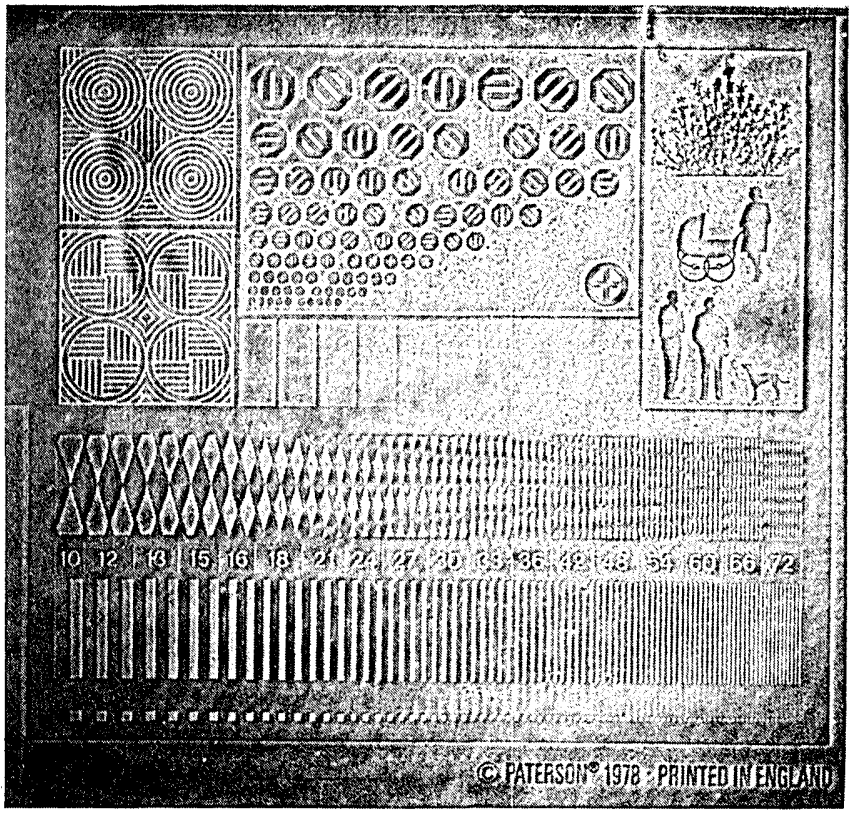

(a)

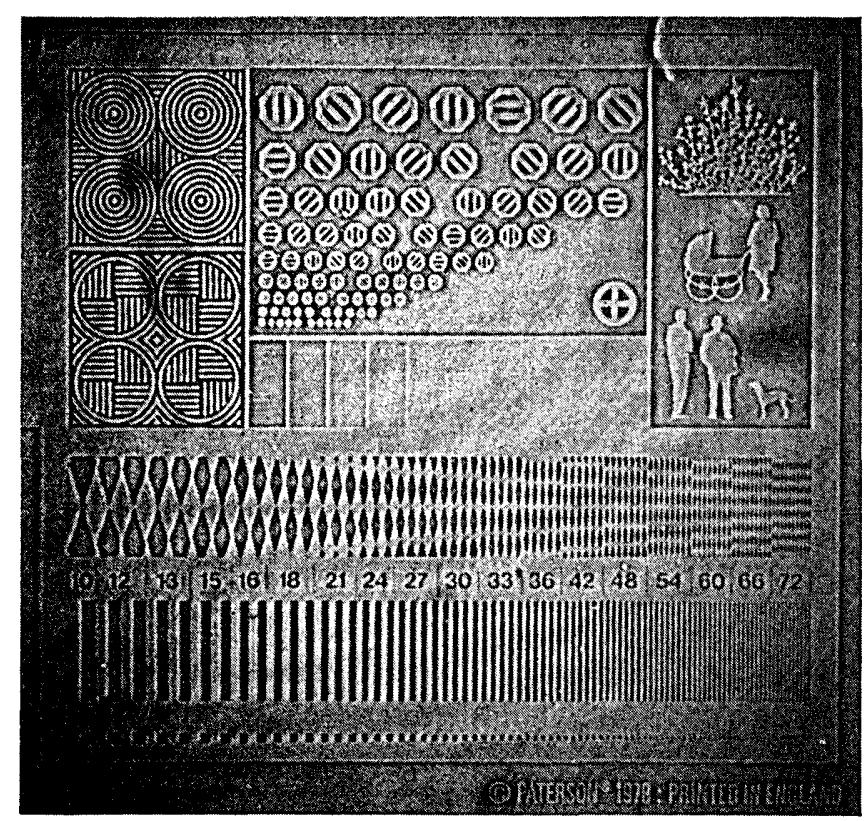

(b)

Fig. 4. Phase contrast: (a) positive, (b) negative.

their phase difference has to be taken into account. Figure 3 displays the dependence of the amplitude and phase-filtering properties of our setup on the analyzer orientation, with the quarter-wave plate omitted. For simplification we assume the electrical field to be zero for the zero-order spot and $7 \mathrm{kV} \mathrm{cm}$ cm $^{-1}$ elsewhere; this is realistic, at least for weak-phase objects, which are usually consid- ered with phase-constrast applications. The three graphs in Fig. 3 represent the amplitude transparence of the selfinduced filter for the zero-order spot, the diffracted light, and the relative phase difference between them. It can also be seen that because of its elliptical polarization, the low-intensity diffracted light cannot be fully blocked without the quarter-wave plate. The direction of the readout 
light polarization was chosen to maximize the ellipicity of the light after passing through the crystal. Because of the optical activity of BSO (the polarization plane of light with $\lambda=633 \mathrm{~nm}$ will be rotated $70^{\circ}$ clockwise by a $3-\mathrm{mm}$ thick BSO crystal), this orientation will not be $45^{\circ}$ to the main axes; the angle has to be chosen in a way that the average angle will be $45^{\circ}$ with respect to the main axes.

\section{APPLICATIONS}

\section{Variable Phase Contrast}

Figure 3 shows a remarkable analyzer position in which the undiffracted light is eliminated and the phase difference shows a discontinuity of $180^{\circ}$, jumping from $-90^{\circ}$ to $+90^{\circ}$. This is similar to the well-known Zernike phasecontrast method, which is used to convert weak phase dif- ferences into amplitude modulations. ${ }^{15}$ In comparison with a fixed phase plate, a variable amplitude suppression of the zero-order spot is more feasible. From this point of view the setup is similar to the polanret system ${ }^{16}$ or the Wolter method. ${ }^{17}$ Examples of positive and negative phase contrast are shown in Fig. 4. Switching between phase contrast and bright-field imaging is made possible by turning the applied voltage on and off.

\section{Edge Enhancement}

Choosing an amplitude transmission of $T=0$ for the zero spot, we will get a dark-field image that leads to edge enhancement. Each edge is transformed into a bright double line with a small central dip located at the edge position, which can be used for precise linewidth measurements. ${ }^{18,19}$

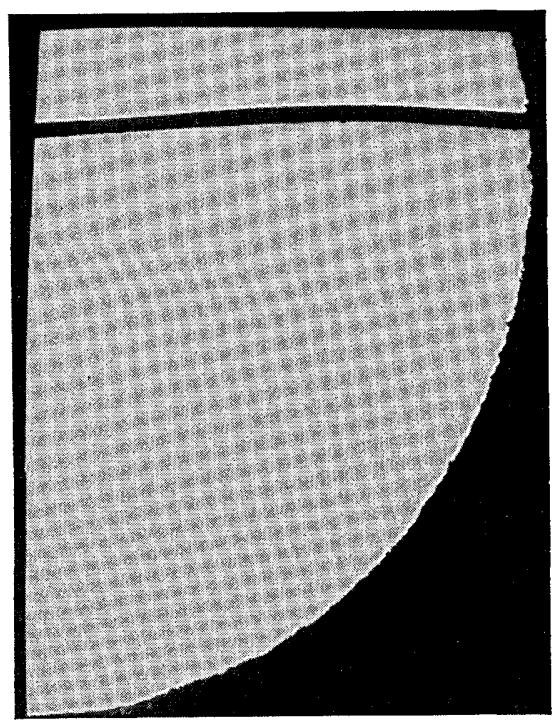

(a)

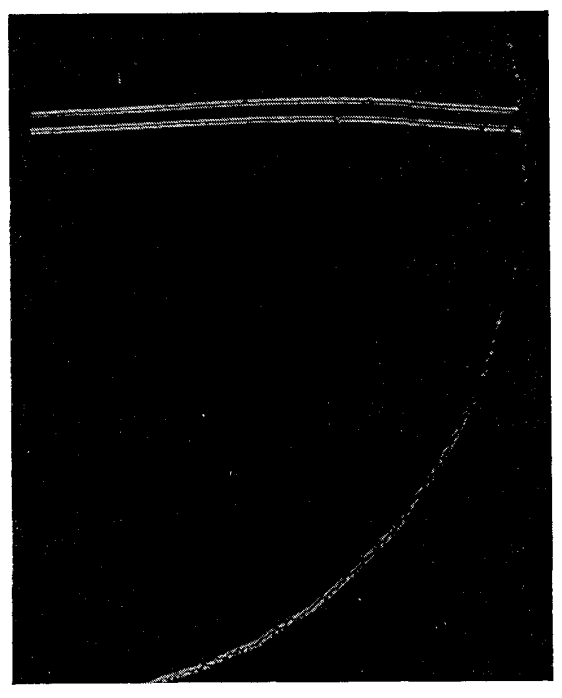

(b)

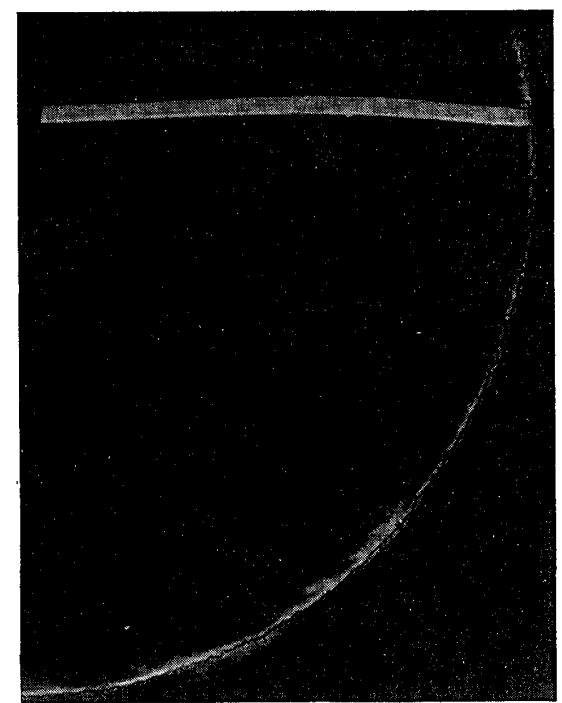

(c)

Fig. 5. Edge enhancement: (a) input image of a wire; (b) edge-enhanced and (c) contrast-inverted images.

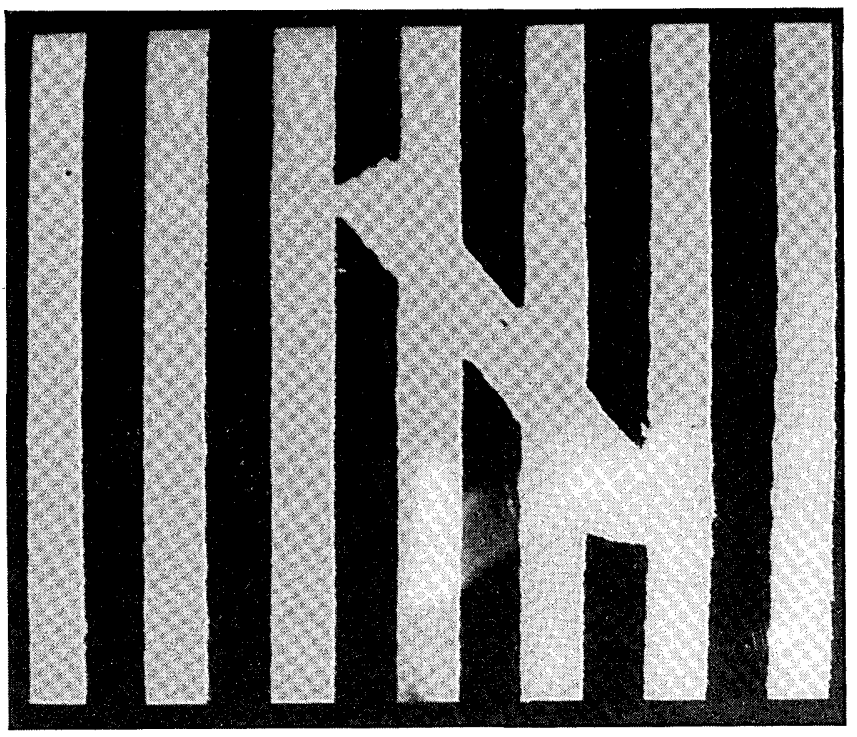

(a)

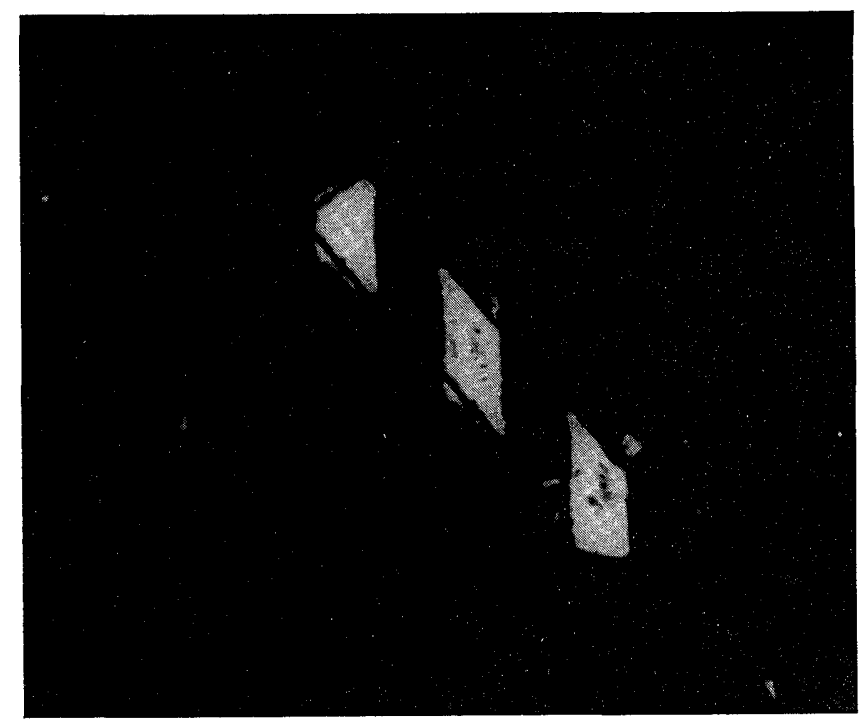

(b)

Fig. 6. Defect enhancement: (a) input image with disturbance in periodicity, (b) defect-enhanced image. 


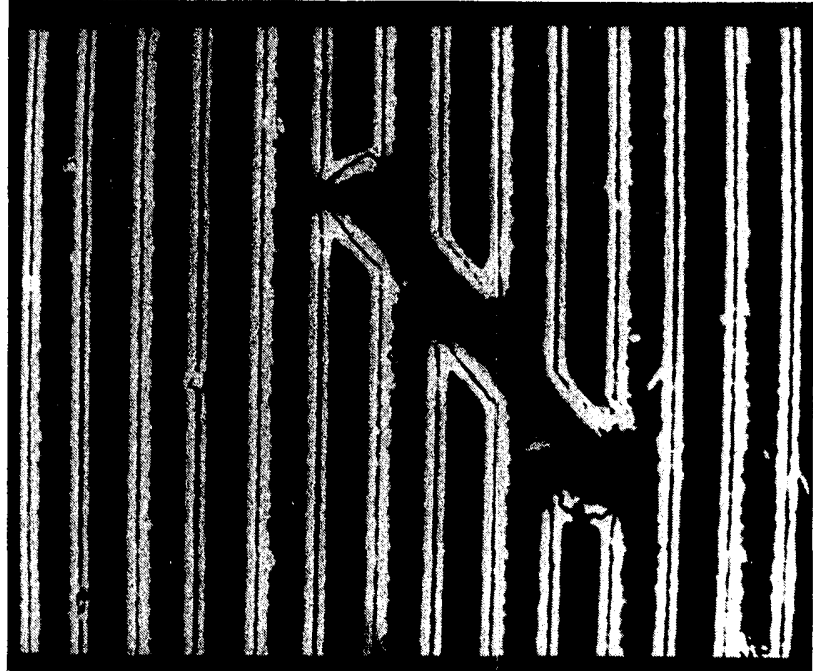

Fig. 7. Edge-enhanced periodic image of Fig. 6(a).

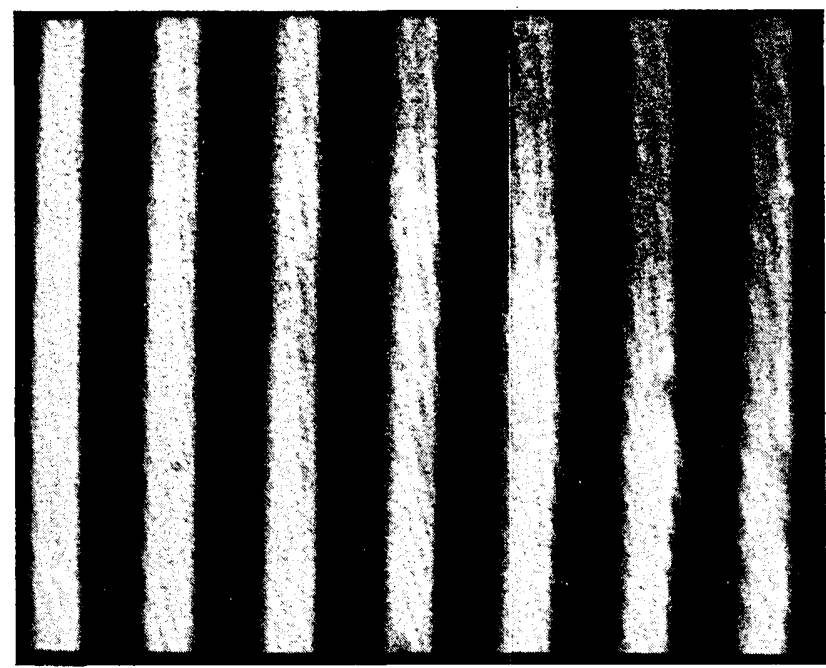

Fig. 8. Defect suppression applied to Fig. 6.

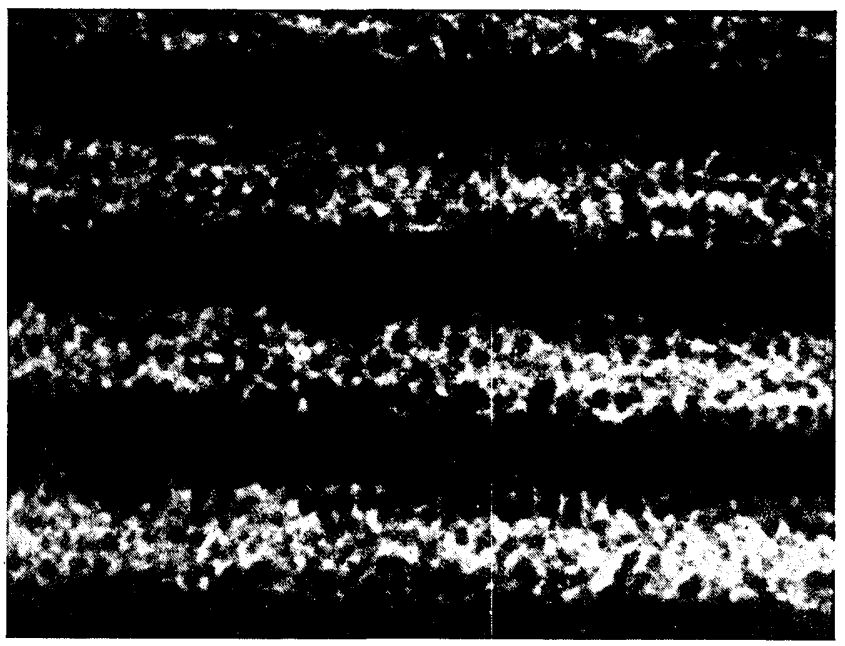

(a)

Fig. 9. Example of noise suppression:
The transfer function of this system approximates a Gaussian-shaped high-pass filter, which in contrast to a steplike annular stop shows a point-spread function without oscillating sidelobes. The effective diameter of the high-pass filter can be dynamically varied by using homogeneous background illumination to enhance the overall conductivity of the BSO crystal. As expected theoretically, the edge-enhanced image of a small wire $(90 \mu \mathrm{m}$ in diameter) then becomes a contrast-inverted image, because the original image can be thought of as an average amplitude (which is represented by the zero-order spot), to which the wire-shaped image with opposite amplitude sign is added. Suppressing exactly the zero spot should therefore result in contrast inversion, which indeed can be observed (Fig. 5).

The possibility of changing the sharpness of the filter spot is also important for the phase-contrast applications: The smaller the zone-plate spot, the greater the area of the extended objects, which can be displayed with constant contrast.

\section{Defect Enhancement}

Difficulties arise when edge enhancement is applied to periodic objects: Because of their discrete spectral orders all their spectra will be treated like the zero-order spot. This leads to the powerful application of this phenomenon for defect enhancement in periodic structures by eliminating all diffraction peaks; only the nonperiodic structures, such as disturbances in the periodicity and dust particles, will remain in the output image. In contrast to conventional filtering techniques, this method is again adaptable to different objects, being-within certain limits, of course-scale, shift, and rotation invariant.

An example of defect enhancement is given in Fig. 6. As in phase-contrast applications, it is sometimes useful to apply a uniform background illumination in order to increase the overall conductivity, which results in sharpening of the suppressed peaks and therefore in larger (in this case defect-enhanced) areas.

The preliminary results can lead to the following interesting applications:

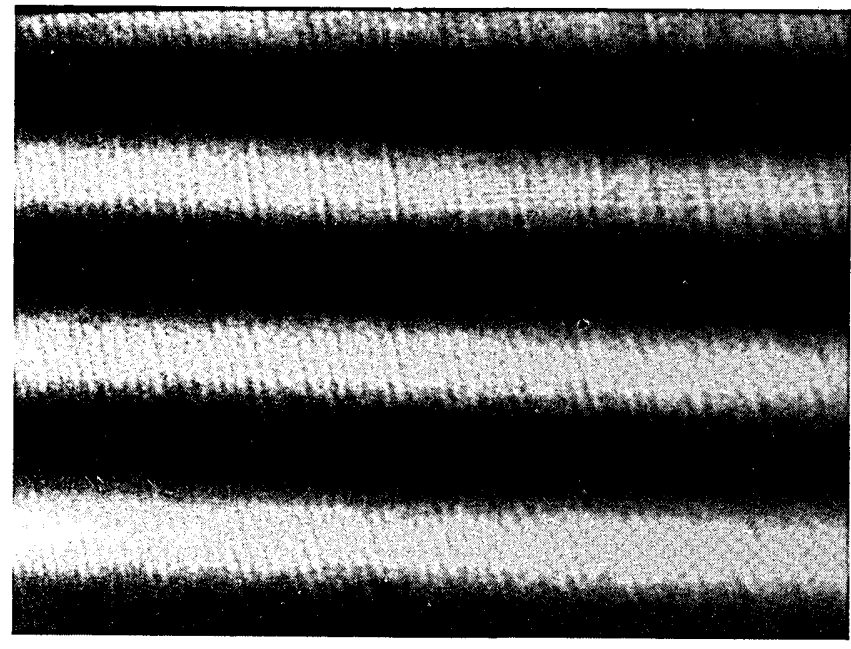

(b)

(a) noisy input, (b) restorated grating. 
Detection of dust and scratches on periodic structures, e.g., memory cells on digital circuits;

Quality testing of lithography masks, digital LCD arrays, etc.

Automatic production surveillance in textile fabrication.

With a slight magnification of the point-source diameter it is also possible to perform edge detection on periodic structures; Fig. 7 was obtained by slightly defocusing the rotating diffusor, which led to the necessary decrease in the grating diffraction peaks so that they could be biased by the uniform background illumination. Then it was possible to suppress only the lower-order diffraction peaks for edge enhancement.

\section{Noise Suppression}

The filter process that is the inverse of the defect enhancement described above permits the restoration of gratings by removal of the elliptically polarized low-intensity defect spectra. Also in this case biasing of the nonperiodic spectral parts is necessary because the combination of a quarter-wave plate and an analyzer is able to block only one certain birefringence and therefore one certain conductivity of the BSO at a time. Since suppression of all nonperiodic spectral contributions must be obtained, the overall conductivity has to be increased in order to keep the birefringence constant outside the diffraction peak areas. For Fig. 8 we applied defect suppression to the object in Fig. 6(a), resulting in a remarkable grating restoration. An application of noise suppression is shown in Fig. 9.

\section{CONCLUSIONS}

We have demonstrated a new adaptive self-addressed spatial filtering technique with photorefractive BSO crystals, which is able to perform different applications such as Zernike phase contrast, edge enhancement, defect enhancement, and noise suppression within a single device. By means of ellipticity selection by polarization compensation, we have realized a high- (or low-) intensity-pass filter, which acts also, depending on the frequency spectrum of the object, as a low- or high-pass filter. The tunable physical quantities of the device are (in addition to the mentioned compensation) the voltage applied to the crystal, the strength of the incoherent background illumination, and the spatial coherence of the laser-light source.

All results presented in this paper were obtained by using one simple nonholographic image-processing setup, which is insensitive to vibrations and, within certain lim- its, is rotation, shift, and scale invariant. The filter functions can be updated within a fraction of a second.

Because of the time delay in the filter-generation process, it should be possible to realize a novelty filter that detects scale and rotational changes of gratings or other periodical structures.

\section{REFERENCES}

1. J. P. Huignard and F. Micheron, "High-sensitivity read-write volume holographic storage in BSO and BGO crystals," Appl. Phys. Lett. 29, 591-593 (1976).

2. P. Günter, "Holography, coherent light amplification and optical phase conjugation with photorefractive materials," Phys. Rep. 93, 199-299 (1982).

3. R. A. Sprague and P. Nisenson, "The PROM-a status report," Opt. Eng. 17, 256-266 (1978).

4. F. Höller and H. J. Tiziani, "A spatial light modulator using BSO crystals," Opt. Commun. 58, 20-24 (1986).

5. L. Pugliese and G. M. Morris, "Bandpass filtering in $\mathrm{BaTiO}_{3}$," Appl. Opt. 27, 4535-4539 (1988).

6. J. Ma, L. Liu, Z. Wang, and L. Xu, "Controllable real-time simple spatial filter based on selectivity erasing in photorefractive two-beam coupling," Opt. Commun. 74, 15-18 (1989).

7. J. A. Khoury, G. Hussian, and R. W. Eason, "Contrast manipulation and controllable spatial filtering via photorefractive two-beam coupling," Opt. Commun. 70, 272-276 (1989).

8. N. A. Vainos and R. W. Eason, "Real time edge enhancement by active spatial filtering via five wave mixing in photorefractive BSO," Opt. Commun. 59, 167-172 (1986).

9. J. P. Huignard and J. P. Herriau, "Real-time coherent object edge reconstruction with BSO crystals," Appl. Opt. 17, 26712672 (1978).

10. E. Ochoa, J. W. Goodman, and L. Hesselink, "Real-time enhancement of defects in a periodic mask using photorefractive BSO," Opt. Lett. 10, 430-432 (1985).

11. R. C. Jones, "A new calculus for the treatment of optical systems," J. Opt. Soc. Am. A 38, 671 (1948).

12. G. N. Ramachandran and S. Ramaseshan, "Crystal optics," in Encyclopaedia of Physics, S. Flügge, ed. (Springer-Verlag, Berlin, 1961), Vol. 25, p. 1.

13. P. Pellat-Finet, "Measurement of the electro-optical coefficient in BSO," Opt. Commun. 50, 275-280 (1984).

14. D. Joyeux and S. Lowenthal, "Optical Fourier transform: what is the optimal setup?" Appl. Opt. 21, 4368-4372 (1982).

15. H. Bayer, Theorie und Praxis des Phasenkontrastverfahrens (Akademische Verlagsgesellschaft, Frankfurt, 1965).

16. M. Pluta, "Simplified polanret system for microscopy," Appl. Opt. 28, 1453-1466 (1989).

17. H. Wolter, "Phasen-und Amplitudenmessung durch Elliptizitätsanalyse an Phasenkontrastbildern," Z. Phys. 140, 57-74 (1955).

18. K. G. Birch, "A spatial frequency filter to remove zero frequency," Opt. Acta 15, 113-127 (1968).

19. M. Young, "Spatial filtering for linewidth measurement," Appl. Opt. 28, 1467-1473 (1989). 